\title{
Sensitivity and specificity of the antigen-based anterior nasal self-testing programme for detecting SARS-CoV-2 infection in schools, Austria, March 2021
}

Peter Willeit ${ }^{1,2,{ }^{*}}$, Benoît Bernar ${ }^{3,{ }^{*},}$, Christoph Zurl $4,5,6,{ }^{*}$, Mariam Al-Rawij,8, Andrea Berghold9, David Bernhard ${ }^{10,11}$, Wegene

Borena $^{12}$, Christian Doppler ${ }^{10,11}$, Reinhold Kerbl ${ }^{13}$, Alwin Köhler7,8, Robert Krause ${ }^{4,5}$, Bernd Lamprecht ${ }^{14}$, Johannes Pröll ${ }^{10}$, Hannes Schmidt ${ }^{7,15}$, Ivo Steinmetz ${ }^{16}$, Evelyn Stelz ${ }^{16}$, Heribert Stoiber ${ }^{12}$, Dorothee von Laer ${ }^{12}$, Johannes Zuber ${ }^{7,17}$, Thomas Müller ${ }^{3}$, Volker

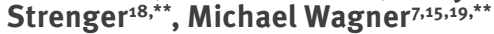

1. Clinical Epidemiology Team, Department of Neurology, Medical University of Innsbruck, Innsbruck, Austria

2. Department of Public Health and Primary Care, University of Cambridge, Cambridge, United Kingdom

3. Department of Pediatrics I, Medical University of Innsbruck, Innsbruck, Austria

4. Section of Infectious Diseases and Tropical Medicine, Department of Internal Medicine, Medical University of Graz, Graz, Austria

5. BioTechMed-Graz, Graz, Austria

6. Department of Paediatrics and Adolescent Medicine, Division of General Paediatrics, Medical University of Graz, Graz, Austria

7. Vienna Covid-19 Detection Initiative, Vienna, Austria

8. Max Perutz Labs, University of Vienna, Vienna, Austria

9. Institute for Medical Informatics, Statistics and Documentation, Medical University of Graz, Graz, Austria

10. Center for Medical Research, Faculty of Medicine, Johannes Kepler University Linz, Linz, Austria

11. Division of Pathophysiology, Institute of Physiology and Pathophysiology, Johannes Kepler University Linz, Linz, Austria

12. Institute of Virology, Medical University Innsbruck, Innsbruck, Austria

13. Department of Paediatrics and Adolescent Medicine, LKH Hochsteiermark, Leoben, Austria

14. Department of Pulmonology, Kepler-University-Hospital, Faculty of Medicine, Johannes Kepler University Linz, Linz, Austria

15. Centre for Microbiology and Environmental Systems Science, Department of Microbiology and Ecosystem Science, University of Vienna, Vienna, Austria

16. Diagnostic and Research Institute of Hygiene, Microbiology and Environmental Medicine, Medical University of Graz, Graz, Austria

17. IMP - Research Institute of Molecular Pathology, Vienna, Austria

18. Department of Paediatrics and Adolescent Medicine, Division of Pediatric Pulmonology and Allergology, Medical University Graz, Graz, Austria

19. Center for Microbial Communities, Department of Chemistry and Bioscience, Aalborg University, Aalborg, Denmark

* These authors contributed equally as first authors.

** These authors contributed equally as senior authors.

Correspondence: Volker Strenger (volker.strenger@medunigraz.at)

Citation style for this article:

Willeit Peter, Bernar Benoît, Zurl Christoph, Al-Rawi Mariam, Berghold Andrea, Bernhard David, Borena Wegene, Doppler Christian, Kerbl Reinhold, Köhler Alwin, Krause Robert, Lamprecht Bernd, Pröll Johannes, Schmidt Hannes, Steinmetz Ivo, Stelzl Evelyn, Stoiber Heribert, von Laer Dorothee, Zuber Johannes, Müller Thomas, Strenger Volker, Wagner Michael. Sensitivity and specificity of the antigen-based anterior nasal self-testing programme for detecting SARS-CoV-2 infection in schools, Austria, March 2021. Euro Surveill. 2021;26(34):pii=2100797. https://doi.org/10.2807/1560-7917.ES.2021.26.34.2100797

This study evaluates the performance of the antigenbased anterior nasal screening programme implemented in all Austrian schools to detect SARS-CoV-2 infections. We combined nationwide antigen-based screening data obtained in March 2021 from 5,370 schools (Grade 1-8) with an RT-qPCR-based prospective cohort study comprising a representative sample of 244 schools. Considering a range of assumptions, only a subset of infected individuals are detected with the programme (low to moderate sensitivity) and noninfected individuals mainly tested negative (very high specificity).

Early recognition and isolation of infected individuals are crucial in containing the coronavirus disease (COVID-19) pandemic. Rapid antigen tests deliver timely results, can be performed without laboratory resources, and are widely used in screening programmes for severe acute respiratory syndrome coronavirus 2 (SARS-CoV-2) [1-3]. As transmission and outbreaks occur in educational settings [4], a nationwide screening programme was introduced at Austrian schools in January 2021 that implemented regular antigen-based anterior nasal self-tests for pupils, teachers and administrative staff [5].

The present study aimed to evaluate the performance of the antigen-based screening programme for detecting SARS-CoV-2 infections in Austrian schools. The design of the study is outlined in Figure 1. To provide context, the mean 7-day community incidence during the study period was 198 per 100,000 individuals across all ages.

\section{Nationwide rapid antigen test-based screening programme}

The first component used aggregate data from the nationwide rapid antigen test-based screening programme (Figure 1) provided by the Federal Ministry of Education, Science and Research. Data were collected in Weeks 9-11 at primary schools (Grades 1-4) and lower secondary schools (Grades 5-8) and involved 680,620 pupils and 104,087 teachers and administrative staff from 5,370 schools. Participants 
were typically asymptomatic or pauci-symptomatic since pupils and teachers with symptoms were strictly advised to stay at home. Participation in the screening programme was free of charge, obligatory for in-person teaching, and required written informed consent. Participants conducted self-testing under the supervision of a teacher, twice per week in primary schools (Mondays, Wednesdays) and once per week in lower secondary schools (Mondays or Wednesdays as

\section{FIGURE 1}

Study design for evaluating the performance of the rapid antigen test-based SARS-CoV-2 screening programme in schools, Austria, March 2021

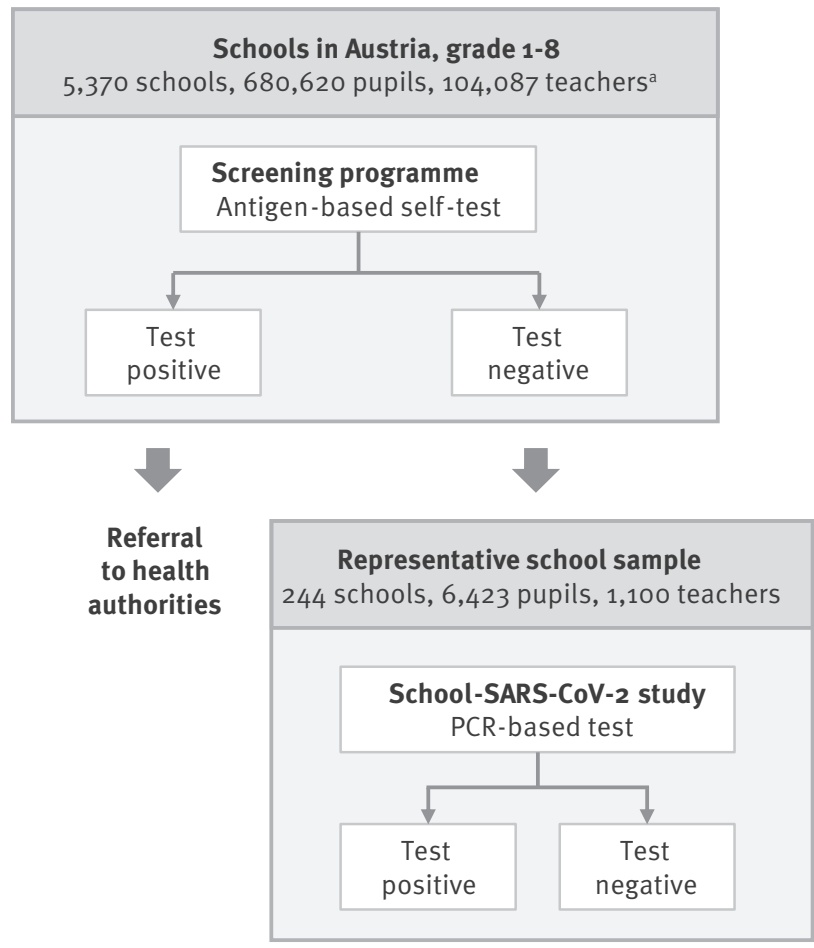

a Included administrative staff. classes were split in two fixed cohorts and taught in school in a staggered manner). Mean participation rate over Weeks 9-11 was 98.5\% (range: 96.4-98.8).

The screening programme used two rapid antigen tests licenced for anterior nasal self-testing (Lepu Medical SARS-CoV-2 Antigen Rapid Test, Lepu Medical Technology, Beijing, China [6]; and Flowflex SARSCoV-2 Antigen Rapid Test, ACON Biotech, Hangzhou, China [7]). The mean percentage tested positive each week is provided in the Table. Positively tested individuals were sent home immediately and referred to the local health authorities as suspected cases for further testing.

\section{Austrian School-SARS-CoV-2 study}

The second component of this study used data from the Austrian School-SARS-CoV-2 study (Figure 1), a prospective cohort study involving pupils and teachers from a representative sample of Austrian schools (Grade 1-8). Every 3-5 weeks during periods of the school year 2020/21 not affected by school closures, participants were tested for SARS-CoV-2 infection using gargling, pooling and in-house RT-qPCR analysing multiple target genes (i.e. ORF1b, ORF10 and N, the latter being detected by two different assays $\mathrm{N}_{1}$ and N2). For all positive samples, at least two viral genes were detected. In addition, an RT-qPCR test for human RNA was included in all assays. The RT-qPCR controls included RNA extracts (i) from a previously tested positive sample with a target $\mathrm{Cq}$ value of 25 or synthetic SARS-CoV-2 RNA control $(1 \times 104$ molecules per reaction; Twist Biosciences, San Francisco, United States), (ii) from a previously tested negative sample and (iii) a no-template control. Further details on study design and methodology have been published previously [8].

Our analysis focused on Round 3 of the Austrian SchoolSARS-CoV-2 study, which was conducted between 1 and 18 March 2021 (Weeks 9-11) and included 6,423 pupils and 1,100 teachers (from 244 schools) who had

TABLE

Number of participants and percentage ${ }^{a}$ tested positive, SARS-CoV-2 screening studies in schools, Austria, March 2021 $(\mathrm{n}=784,707)$

\begin{tabular}{|l|c|c|c|c|}
\hline \multirow{2}{*}{} & \multicolumn{2}{c|}{$\begin{array}{c}\text { Antigen-based screening } \\
\text { programme }\end{array}$} & \multicolumn{2}{c|}{ School-SARS-CoV-2 study } \\
\cline { 2 - 5 } & Participants (n) & $\%$ positive ${ }^{\mathrm{a}}$ & Participants (n) $^{\mathrm{b}}$ & $95 \% \mathrm{Cl}$ \\
\hline Pupils in primary schools & 360,948 & 0.106 & 4,161 & 0.26 \\
\hline Pupils in lower secondary schools & 319,672 & 0.063 & 2,262 & $0.13-0.52$ \\
\hline Teachers at primary/lower secondary schools & $104,087^{\mathrm{c}}$ & 0.292 & 1,100 & $0.04-0.41$ \\
\hline
\end{tabular}

$\mathrm{Cl}$ : confidence interval.

a For the antigen-based screening programme, the percentage tested positive was the mean percentage of participants tested positive each week weighted to the number of participating individuals. The percentages positive are provided without $95 \% \mathrm{Cl}$ because all pupils and educational staff were involved in the programme, rather than drawing a representative sample. The percentages of participants tested positive in Weeks 9, 10 and 11 were $0.088,0.090$ and 0.140 among pupils in primary schools, $0.055,0.053$ and 0.079 among pupils in lower secondary schools and $0.309,0.313$ and 0.254 among teachers and administrative staff in primary/lower secondary schools.

${ }^{\mathrm{b}} 95 \% \mathrm{Cl}$ of prevalence estimates in the Austrian School-SARS-CoV-2 study were calculated from robust standard errors estimated based on clustered Sandwich estimators.

c Included administrative staff.

All participants in the School-SARS-CoV-2 study had before the study tested negative in the antigen-based screening programme. 
previously tested negative in the rapid antigen testbased screening programme. Key findings on the prevalence of RT-qPCR-detected SARS-CoV-2 infection are shown in the Table. Overall prevalence did not differ significantly by time since the last antigen-based selftest: Prevalence was $0.27 \%(8 / 2,985)$ in children and teachers with a negative self-test on the same day vs $0.18 \%(8 / 4,538)$ for people with earlier rapid antigen testing ( $85 \%$ tested on the preceding day), corresponding to an odds ratio of 1.51 (95\% confidence interval: $0.53-4.37 ; p=0.435)$. There was no significant difference in $\mathrm{Cq}$ values among positively tested individuals in Round 3 compared with earlier rounds when no antigen-based screening programme had been in place (Figure 2). Of note, eight of 14 pupils and both teachers who tested positive by RT-qPCR had $\mathrm{Cq}$ values $>30$ for all tested targets.

\section{Estimated sensitivity and specificity of the Austrian antigen-based screening programme}

We modelled sensitivity and specificity of the antigen-based screening programme at Austrian schools by (i) combining findings from the two data sources, (ii) assuming a range of positive predictive values for the rapid antigen test (20-100\%) and (iii) using point estimates and 95\% confidence interval limits in the Austrian School-SARS-CoV-2 study as estimates of the negative predictive value (Figure 3 ). For instance, assuming a positive predictive value of $20 \%$, sensitivity is expected in the range of $3.9-13.6 \%$ among pupils in primary schools, $3.0-22.6 \%$ among pupils in lower secondary schools and $7.4-56.5 \%$ among teachers. Corresponding sensitivity ranges are 10.9-32.0\%, 8.4$46.7 \%$ and $19.4-79.6 \%$ assuming a positive predictive value of $60 \%$, and $17.0-44.0 \%, 13.3-59.4 \%$ and $28.7-$ $86.6 \%$ assuming a perfect positive predictive value of $100 \%$. The model indicated high specificity values at $20 \%, 60 \%$ and $100 \%$ assumed positive predictive values of $99.92 \%, 99.6 \%$ and $100 \%$ for pupils in primary schools, $99.95 \%, 99.7 \%$ and $100 \%$ for pupils in lower secondary schools and $99.77 \%, 99.88 \%$ and $100 \%$ for teachers at primary/secondary schools.

\section{Ethical statement}

Ethical approval was not required for the data from the nationwide rapid antigen test-based screening programme because we used aggregate data only. The Austrian School-SARS-CoV-2 study received ethics approvals by the ethics committees of the Medical University of Graz (no. 32-672 ex 19/20), Medical University of Innsbruck (no. 1319/2020), the Johannes

\section{FIGURE 2}

Cq values of unpooled RT-qPCR of positively tested participants in Round 3 compared with Rounds 1 and 2, School-SARSCoV-2 study, Austria, March 2021 ( $\mathrm{n}=92$ in Rounds 1 and 2, $\mathrm{n}=16$ in Round 3)

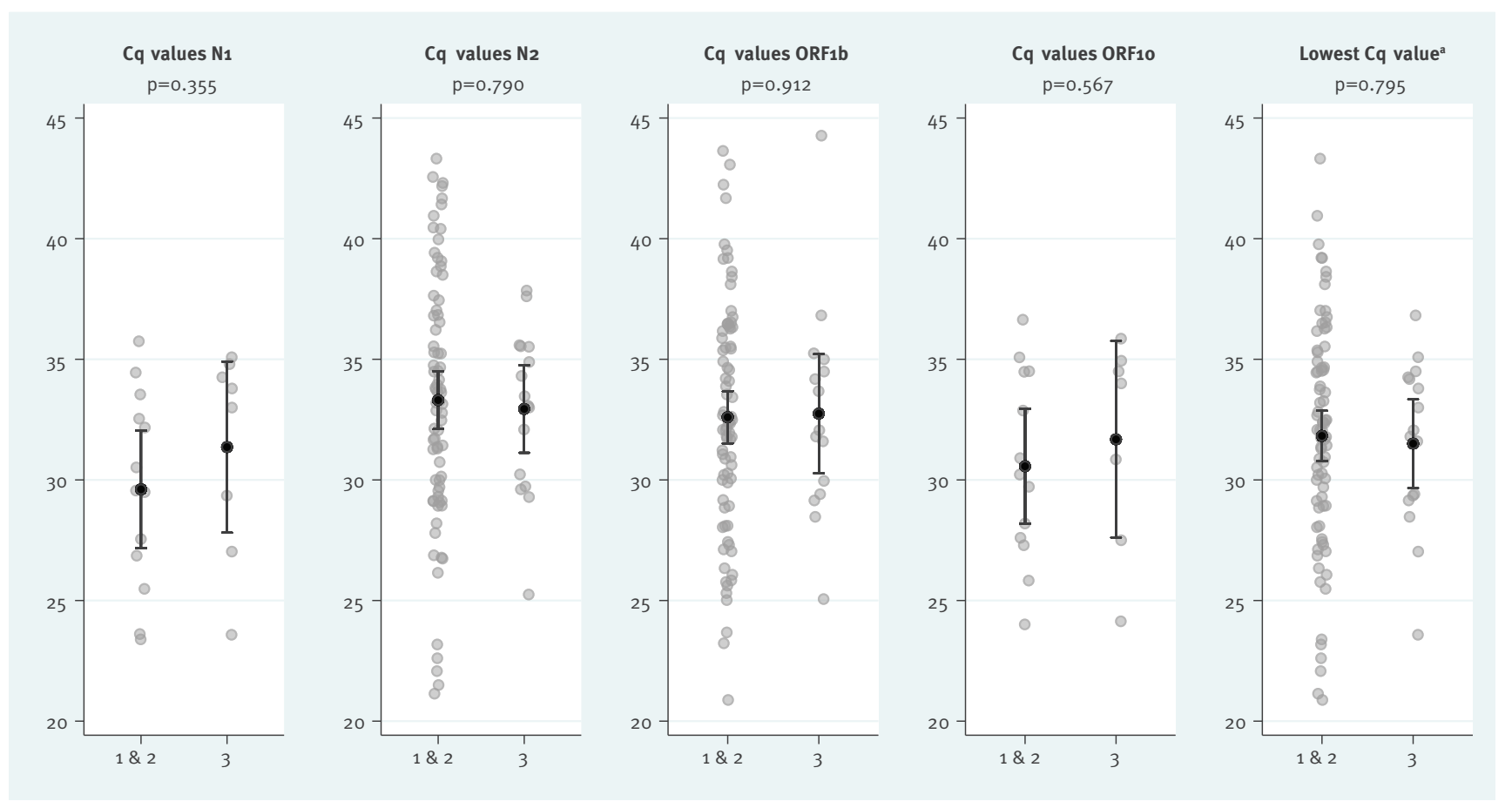

Round of examination

\footnotetext{
${ }^{a}$ Lowest value among the different Cq values of the RT-qPCR target genes.

Grey circles: Cq values of the individual RT-qPCR positive cases; black dots and vertical lines: their arithmetic means and $95 \%$ confidence intervals. N, ORF1b, and ORF10 are RT-qPCR target genes; N1 and N2 represent two different RT-qPCR assays for detection of the N-gene. A two-sample t-test was used to test for difference in Cq values between positively tested participants at Round 1 and 2 compared with positively tested participants at Round 3. Round 1 took place from 28 September to 22 October 2020 (40 RT-qPCR positive cases), Round 2 from 10 to 16 November 2020 (52 positive cases) and Round 3 from 1 to 18 March 2021 (16 positive cases).
} 
Kepler University of Linz (no. 1222/2020), and the University of Vienna (no. 00591/2020). Participants or their legal representative provided written informed consent.

\section{Discussion}

The present study scrutinised the antigen-based screening programme introduced at Austrian schools in early 2021 to detect SARS-CoV-2 cases. While selfcollected anterior nasal swabs can be easily performed, were well accepted by the children in our study and help increase testing capacities, knowledge about their performance in real-world settings are required to guide further decision-making.

While several studies exist on sensitivity and specificity of rapid antigen tests in adults [2], a recent Cochrane review identified a lack of large-scale data on the performance of serial antigen-based screening strategies in asymptomatic school-aged children [3]. Across various modelled scenarios, we observed lower sensitivity but similar specificity in children compared with prior studies in adults [3]. Collection of specimens is not performed by trained medical staff but by pupils in Grades 1-8 and their teachers, which might substantially impact the detection rate. Furthermore, sensitivity of antigen-based tests is highest in symptomatic patients, in whom viral loads are higher $[9,10]$. Less is known about viral loads in the anterior nasal region compared with the throat in asymptomatic people and whether age-specific differences exist, but some data indicate that virus presence in the anterior nose can be delayed in asymptomatic people, leading to a lower detection rate in the early phase of infection $[11,12]$. Consequently, an a priori reduced sensitivity can be assumed in our cohort of asymptomatic individuals. Indeed, many individuals who falsely tested negative in the antigen-based screening programme had $\mathrm{Cq}$ values $>30$ at the moment of testing. While this may indicate a lower viral load and infectivity at this stage, $\mathrm{Cq}$ trajectories in positive cases over time are unclear, and some cases in the early phase of infection might have subsequently developed lower $\mathrm{Cq}$ values.

Our study has strengths and limitations. By combining nationwide data on the antigen-based screening programme with RT-qPCR testing in a carefully chosen representative sample of schools, we provide a unique insight into the potential and limitations of one of the key measures to mitigate SARS-CoV-2 transmission in

\section{FIGURE 3}

Modelled sensitivity of antigen-based anterior nasal self-testing programme assuming a range of positive predictive values and point estimates of the negative predictive value detected in the School-SARS-CoV-2 study, Austria, March 2021
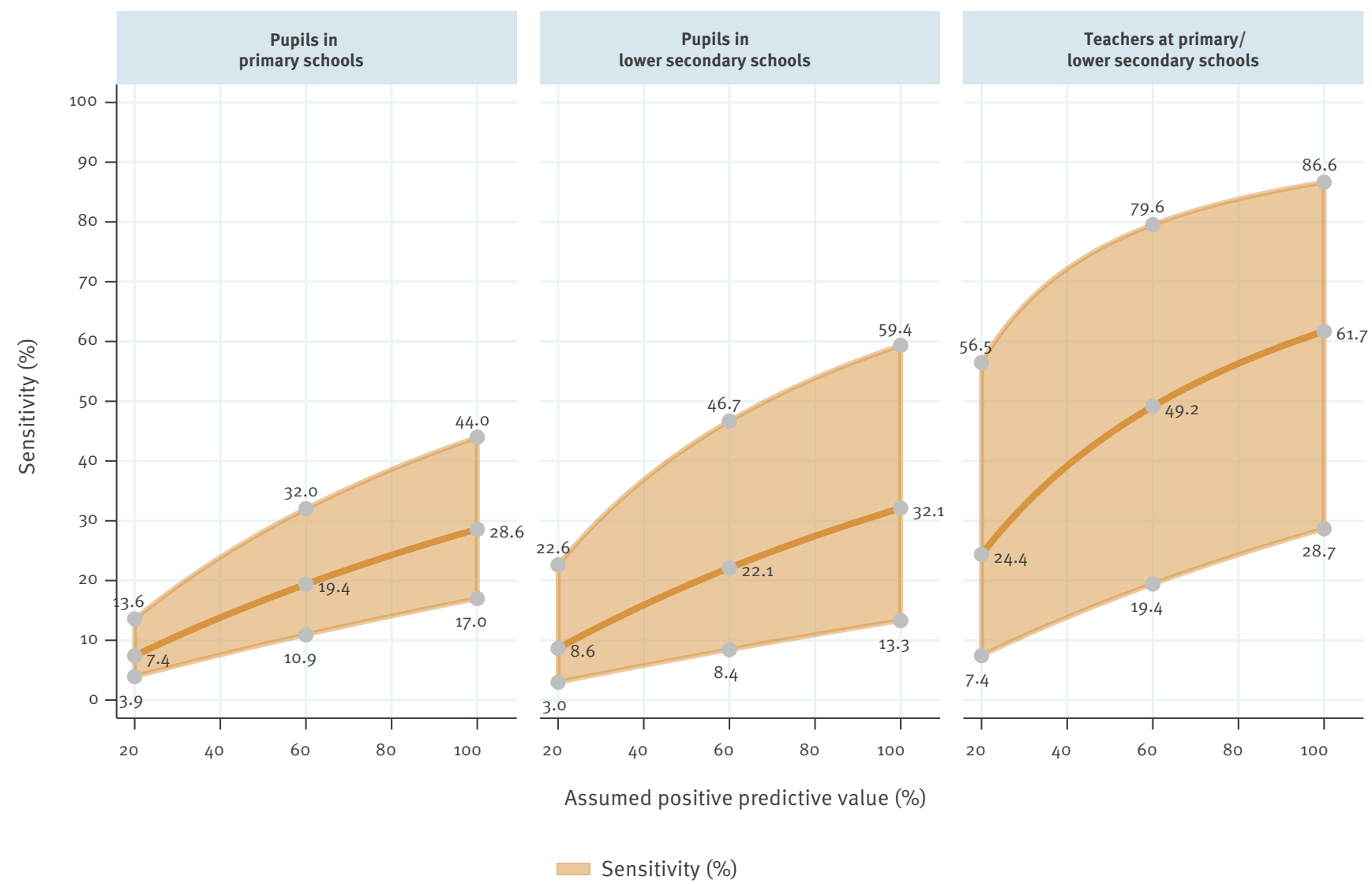

Sensitivity (\%)

Dark orange: point estimates; light orange areas: $95 \%$ confidence interval limits. The numbers next to the grey circles are exemplary sensitivity values at positive predictive values of $20 \%, 60 \%$ and $100 \%$. 
schools. However, our findings may not be generalisable to other countries and antigen tests. Also, for reasons of data protection, only aggregate data were available from the screening programme, precluding more detailed analyses. Furthermore, health authorities could not provide data on how frequently a positive rapid antigen test was confirmed with RT-qPCR. To account for this limitation, our modelling involved a range of positive predictive values.

\section{Conclusion}

Our study indicates that only a subset of infected individuals are detected with the antigen-based screening programme at Austrian schools (low to moderate sensitivity). Non-infected individuals were largely tested negative (very high specificity). Given the low-tomoderate sensitivity of antigen-based anterior nasal self-testing particularly in children, additional measures such as face masks or ventilation are important to prevent secondary cases, especially in periods with high incidence. Furthermore, as a mitigation measure, switching SARS-CoV-2 screening in schools to RT-qPCR based approaches should be preferred, where logistically feasible.

\section{Acknowledgements}

The Austrian School-SARS-CoV-2 Study was funded by the Federal Ministry of Education, Science and Research of the Republic of Austria. Special thanks are due to the technical staff of the collaborating laboratories.

\section{Conflict of interest}

BioTechMed-Graz is a research alliance of three Austrian university to promote inter-university research activities. All authors declare that they have no conflict of interest in relation to the article.

\section{Authors' contributions}

PW, RK, TM, VS and MW designed the study. RK, JZ, AK, DVL, HS, IS and MW planned the logistics and/or laboratory measurements for the study. ES, HS, DB, WB, CD and JP performed laboratory measurements. $\mathrm{PW}, \mathrm{BB}$, and $\mathrm{CZ}$ performed the statistical analysis. PW, BB, CZ, TM, VS and MW drafted the manuscript. MA-R planned the logistics and/or laboratory measurements for the study. $B L$ designed the study. $A B$ advised on the statistical analysis. MA-R, BL and $A B$ also critically revised the manuscript and agreed to be accountable for all aspects of the work. All authors critically revised the manuscript and agreed to be accountable for all aspects of the work.

\section{References}

1. Mina MJ, Andersen KG. COVID-19 testing: One size does not fit all. Science. 2021;371(6525):126-7. https://doi.org/10.1126/ science.abe9187 PMID: 33414210

2. Mina MJ, Peto TE, García-Fiñana M, Semple MG, Buchan IE. Clarifying the evidence on SARS-CoV-2 antigen rapid tests in public health responses to COVID-19. Lancet. 2021;397(10283):1425-7. https://doi.org/10.1016/S01406736(21)00425-6 PMID: 33609444

3. Dinnes J, Deeks JJ, Berhane S, Taylor M, Adriano A, Davenport $C$, et al. Rapid, point-of-care antigen and molecular-based tests for diagnosis of SARS-CoV-2 infection. Cochrane Database Syst Rev, 2021:3:CD013705. Available from: https://www. cochranelibrary.com/cdsr/doi/10.1002/14651858.CD013705. pub2/full/de https://doi.org/10.1002/14651858.CD013705. pub2 PMID: 33760236

4. Hyde Z. COVID-19, children and schools: overlooked and at risk. Med J Aust. 2020;213(10):444-446.e1. https://doi. org/10.5694/mja2.50823 PMID: 33099775

5. Bundesministerium für Bildung, Wissenschaft und Forschung (BMBWF). Wissenschaftliche Begleitung zum Eintritts-Selbsttest (anterio-nasaler Antigen-Schnelltest) an österreichischen Schulen. [Scientific instructions for the access self-test (anterio-nasal antigen rapid test) at Austrian schools]. Vienna: BMBWF. [Accessed: 30 Mar 2021]. German. Available from: https://www.bmbwf.gv.at/Themen/Forschung/ Aktuelles/BeAntiGenT.html

6. Lepu Medical. SARS-CoV-2 Antigen Rapid Test Kit (Colloidal Gold Immunochromatography). Beijing: Lepu Medical. [Accessed: 30 Mar 2021]. Available from: https:// en.lepumedical.com/products/sars-cov-2-antigen-rapid-testkit-colloidal-gold-immunochromatography

7. ACON. Flowflex SARS-CoV-2 Antigen Rapid Test. San Diego: ACON. Accessed: 31 Mar 2021]. Available from: https://www. aconlabs.com/sars-cov-2-antigen-rapid-test

8. Willeit $P$, Krause $R$, Lamprecht B, Berghold A, Hanson B, Stelzl E, et al. Prevalence of RT-qPCR-detected SARS-CoV-2 infection at schools: First results from the Austrian SchoolSARS-CoV-2 prospective cohort study. Lancet Reg Health Eur. 2021;5:100086. https://doi.org/10.1016/j.lanepe.2021.100086 PMID: 34396360

9. Lindner AK, Nikolai O, Kausch F, Wintel M, Hommes F, Gertler $M$, et al. Head-to-head comparison of SARS-CoV-2 antigendetecting rapid test with self-collected nasal swab versus professional-collected nasopharyngeal swab. Eur Respir J. 2021;57(4):2003961. https://doi.org/10.1183/13993003.039612020 PMID: 33303544

10. Krüttgen A, Cornelissen CG, Dreher M, Hornef MW, Imöhl $M$, Kleines M. Comparison of the SARS-CoV-2 Rapid antigen test to the real star Sars-CoV-2 RT PCR kit. J Virol Methods. 2021;288:114024. https://doi.org/10.1016/j. jviromet.2020.114024 PMID: 33227341

11. Austrian Agency for Health and Food Safety (AGES). Evaluierung von SARS-CoV-2-Antigen-Schnelltests aus anterioren Nasenabstrichen im Vergleich zu PCR an Gurgellösungen oder Nasopharyngealabstrichen. [Evaluation of SARS-CoV-2 rapid antigen tests from anterior nasal swabs compared with PCR on gargle solutions or nasopharyngeal swabs]. Vienna: AGES; 2021. German. Available from: https:// www.ages.at/service/service-presse/pressemeldungen/ evaluierung-von-sars-cov-2-antigen-schnelltests-ausanterioren-nasenabstrichen-im-vergleich-zu-pcr-angurgelloesungen-oder-nasopharyngealabstrichen

12. Savela ES, Winnett A, Romano AE, Porter MK, Shelby N, Akana $R$, et al. SARS-CoV-2 is detectable using sensitive RNA saliva testing days before viral load reaches detection range of lowsensitivity nasal swab tests. medRxiv. 2021.04.02.21254771. https://doi.org/10.1101/2021.04.02.21254771. PMID: 33851180

\section{License, supplementary material and copyright}

This is an open-access article distributed under the terms of the Creative Commons Attribution (CC BY 4.0) Licence. You may share and adapt the material, but must give appropriate credit to the source, provide a link to the licence and indicate if changes were made.

Any supplementary material referenced in the article can be found in the online version.

This article is copyright of the authors or their affiliated institutions, 2021. 